J. Chem. Soc. Nigeria, Vol. 46, No.2, pp 0436 - 0443 [2021]

\title{
CATALYTIC DETOXIFICATION OF AQUEOUS CYANIDE USING COPPER CROWN ELECTROSPUN CHITOSAN NANOFIBER
}

N. O. SANYAOLU ${ }^{1 *}$, A, A, IBIKUNLE ${ }^{1}$, F. A. KAREEM ${ }^{2}$, A. O. OGUNMOYE ${ }^{1}$, S. A. OGUNDARE $^{1}$, O. C. ATEWOLARA-ODULE ${ }^{1}$, A. M. HASHIMI ${ }^{1}$, F. AKINWUNMI ${ }^{3}$, S. ADEWUYI $^{3}$, T. SHITTU ${ }^{3}$, C. O. EROMOSELE ${ }^{3}$, AND N. TORTO $^{4 \#}$

${ }^{1}$ Department of Chemical Sciences, Olabisi Onabanjo University,Ago-Iwoye, Ogun State, Nigeria);

${ }^{2}$ Department of Science Laboratory Technology, School of Science and Technology, Gateway Polytechnic, Saapade, Ogun State;

${ }^{3}$ Chemistry Department, Federal University of Agriculture, Abeokuta; T.S: Dept. of Food Science and Technology, Federal University of Agriculture Abeokuta,Ogun State, Nigeria; N.T:

${ }^{4}$ Department of Chemistry, Rhodes University, Grahamstown 6140, South Africa).

*Corresponding author's email: nurudeen.sanyaolu@oouagoiwoye.edu.ng +2348034258406

"NT Present address: Botswana Institute for Technology Research and Innovation (BITRI) C/o Plot No. 50654, Machel Drive Private Bag 0082, Gaborone, Botswana.

Received 06 March 2021; accepted 28 March 2021, published online 10 April 2021

\begin{abstract}
Owing to the common phenomenon of cyanide toxicity, this study focused on oxidative catalytic detoxification of aqueous cyanide in the presence of copper-crown chitosan nanofiber. To overcome the difficulty in electrospinning chitosan, chitosan-salicylaldehyde was initially synthesized via Schiff base condensation, electrospun, and post neutralised into chitosan nanofiber. The copper-crown nanofiber was prepared using the impregnation method. The nanofiber material was characterized using FT-IR, SEM, TGA and DSC. The observed pseudo first-order rate constant for the catalytic $\mathrm{H}_{2} \mathrm{O}_{2}$ oxidation of aqeous cyanide with copper-crown nanofiber as catalyst was found to be 16 times greater than the oxidation with only copper catalyst. In addition, the reaction was found to be dependent on the size of the copper-crown nanofiber, $\mathrm{pH}$, temperature, and $\mathrm{H}_{2} \mathrm{O}_{2} / \mathrm{CN}^{-}$. The reusability of the copper-crown nanofiber catalyst makes the process economically and potentially viable for commercial application.
\end{abstract}

Key words: chitosan; nanofiber; copper; oxidation; cyanide; detoxification

\subsection{INTRODUCTION}

Cyanide contamination is one of the environmental problems which can be linked to both natural and antropogenic sources. There is a proliferation of compounds either in solid, liquid and gaseous forms containing cyanide moiety which are used industrially in which cyanide acts as the primary toxic agents. [1].

The level of exposure to cyanide determines the extent of the severity of the health effects. Some of the symptoms of acute exposure to cyanide includes tachypnoea, headache, vertigo, lack of motor coordination, weak pulse, cardiac arrhythmias, vomiting, stupor, convulsions and coma [2]. Numerous methods exist for cyanide detoxification, but with some set-backs; biological treatment, oxidative method, sulphur(IV) oxide assisted oxidation, natural degradation, acidification, volatilization, and reneutralization[3].

Cyanide oxidation by hydrogen peroxide has been confirmed to be the most viable option as it avoids the formation of toxic intermediates and does not add to the total dissolved solids [4]. However, it suffers from the long reaction time. The inclusion of heterogenous catalyst has been shown to promote the reaction between cyanide and hydrogen peroxide [5]. The oxidation of dilute aqeous cyanide solution using hydrogen peroxide at room temperature was enhanced by the presence of $\mathrm{Ru} / \mathrm{MgO}$ catalyst [6]. Kitis et al., [5] carried out the oxidative destruction of free cyanide with hydrogen peroxide and copper-impregnated pumice as a heterogeneous catalyst. The peroxide oxidizes free and weakly complexed metal cyanides (i.e. nickel, copper, cadmium, 
and zinc cyanides), forming one or less toxic compounds according to the reaction pathways (Equations 1 and 2) [7].

$$
\begin{aligned}
& \mathrm{CNO}^{-}+2 \mathrm{H}_{2} \mathrm{O} \stackrel{\mathrm{H}_{2} \mathrm{O}_{2}}{\longrightarrow} \mathrm{NH}_{4}^{+}+\mathrm{CO}_{3}^{2-} \\
& \mathrm{CN}^{-}+\mathrm{H}_{2} \mathrm{O}_{2} \rightarrow \mathrm{CNO}^{-}+\mathrm{H}_{2} \mathrm{O}
\end{aligned}
$$

Previously, our group considered the complexing ability of chitosan-ligated metal and ease of coordination of cyanide to form stable complexes [8]. This approach served as a cheap alternative for cyanide removal from waste water. Building on this inherent property of chitosan, the development of a system which can combine both the catalytic oxidative approach and utilizing complexing solid support of a large surface area is highly imperative. Characteristically of nanofibers, they possess a large surface area which makes them an excellent matrix for catalyst support, enabling metal dispersion as small particles and inhibiting the formation of flocs. Chitosan, an optically active biopolymeric ligand with a strong affinity for transition metals and has been used as support for the preparation of heterogeneous catalysts in many forms among which the fibrous form has been reported to be of most importance [9]. Using electrospinning technique to form chitosan nanofiber, the copper-crown fibers were subsequently applied as a new oxidative catalyst for total cyanide detoxification in an aqueous solution. The results of the detoxification efficiency of this composite approach are herein presented.

\subsection{EXPERIMENTAL PROCEDURE}

\subsection{Materials}

Chemicals of pure analytical grade were used for the preparation of all reagents. Chitosan with $90 \%$ degree of deacetylation and high molecular weight $600 \mathrm{kDa}$.

2.2 Preparation of iminochitosan nanofiber

Chitosan $(100 \mathrm{~g})$ was first reacted with 130 $\mathrm{mL}$ of salicylaldehyde to form a chitosan Schiff base by adopting the method of ElTahlawy et al.[10]. A mixture of the iminochitosan and TFA in acetone/dry ice bath was then frozen and later thawed in a water bath at ambient temperature. The solution was left overnight for electrospinning which was carried out using the following conditions; $24 \mathrm{KV}, 0.9 \mathrm{mlh}^{-1}$, $15 \mathrm{~cm}, 1.2 \mathrm{~mm}$ needle, $44.9 \%, 17.3{ }^{\circ} \mathrm{C}$ [11]. High Powered Electron Microscope (Olympus BX51) was used for the preliminary characterization of the electrospun chitosan. Electrospun nanofibrous structures were sputter-coated with gold (Denton Desk-1 Sputter Coater) the morphology of the electrospun nanofibers was determined with Scanning Electron Microscope (SEM; Vega Tescan, USA). A thermogravimetric analyser model TA-2960 was employed to determine thermogravimetric curves using Ca. $10 \mathrm{mg}$ samples of the imminochitosan materials in a dynamic atmosphere, under a dry nitrogen flux, with heating from room temperature to $800{ }^{\circ} \mathrm{C}$ at a heating rate of $10^{\circ} \mathrm{Cmin}^{-1}$. The thermal transitions of the iminochitosan were detected using DSC (TAQ10). The temperature and heat flow were calibrated using standard materials (indium and zinc) at cooling and heating rates of $10{ }^{\circ} \mathrm{C} / \mathrm{min}$. Samples with a typical mass of 3-10 mg were encapsulated in sealed aluminum pans.

\subsection{Preparation of copper-crown nanofiber}

Neutralization of the iminochitosan nanofiber mats was carried out in a $1 \mathrm{molL}^{-1} \mathrm{~K}_{2} \mathrm{CO}_{3}$ aqueous solution for $3 \mathrm{~h}$ at $25{ }^{\circ} \mathrm{C}$. After neutralization, it was washed repeatedly with deionized water until a pH 7 was achieved. The fiber was first dried at ambient temperature for $24 \mathrm{~h}$ in the hood and then at $60{ }^{\circ} \mathrm{C}$ under vacuum for $24 \mathrm{~h}$ [12]. Impregnation method was used to prepare the copper-crown nanofiber catalyst according to Ye et al.[13]. A dried sample (0.05 g) of the post-neutralised and pretreated nanofiber was placed in a glass beaker and a stock solution of $\mathrm{CuSO}_{4}(1 \mathrm{~g} / \mathrm{L})$ was added until all the nanofiber was completely immersed for 30 minutes. The mixture was filtered and the nanofiber was rinsed several times with the copper stock solution and once with water. Finally, samples were dried at room temperature until all moisture was removed and

constant weight achieved. IR spectra of $\mathrm{KBr}$ disc pellets of the nanofiber was recorded on 
a Bulk Scientific, spectro 500 FT-IR spectrometer (East Norway, USA).

2.4. Catalytic oxidation of cyanide

The oxidation reaction was set up in a 250 $\mathrm{mL}$ Erlenmeyer flask mounted on a shaker and covered with aluminum foil to prevent photodegradation of cyanide or peroxide during mixing. The shaker was equipped with a temperature controller and was run at 150 $\mathrm{rpm}$. The flask was initially loaded with the copper-crown nanofiber catalyst $\left(500 \mathrm{mg} \mathrm{g}^{-1}\right.$ ) and then spiked with $50 \mathrm{~mL}$ cyanide $(100$ $\mathrm{ppm})$ from the stock solution. Solution $\mathrm{pH}$ values were adjusted using reagent grade $\mathrm{NaOH}(3 \mathrm{M})$ and/or $\mathrm{HCl}(2 \mathrm{M})$ solutions. The oxidation reaction was initiated by the addition of the hydrogen peroxide (160 $\mathrm{mg} / \mathrm{L}$ ) oxidant. At the selected time intervals range of 0 to 40 mins, aliquot samples were collected and the reaction was quenched using $\mathrm{Na}_{2} \mathrm{SO}_{3}[14]$. The samples were thereafter analyzed for residual cyanide using ninhydrin method [15]. Atomic Absorption spectrophotometer (Tokyo, Japan) was used to determine the concentration of copper in the kinetic experiments. Cyanide was analyzed at $590 \mathrm{~nm}$ wavelength with Shimadzu, UV-1650PC UV-vis Spectrophotometer. A control experiment was set up in parallel in the absence of the nanofiber and all other reaction conditions remained unchanged. All readings were done in triplicates $(\mathrm{n}=3)$.

\subsection{RESULTS AND DISCUSSION}

3.1. Synthesis and characterization of nanofiber products

The chemical modifications of chitosan involving the primary amino functionality along the polymer chain resulted in the formation of imine based chitosan. This is confirmed by the appearance of new $v_{\mathrm{N}=\mathrm{C}}$ around $1625 \mathrm{~cm}^{-1}$ in the IR band of the iminochitosan nanofiber (Fig. 1). Also, Figure 2 shows the IR bands of copper crown nanofiber. The bonding of $\mathrm{Cu}$ (II) ion brings about a noticeable change in the shape of the broad absorption around $150-100 \mathrm{~cm}^{-1}$ suggesting an interaction between the metal ion and the nanofiber.

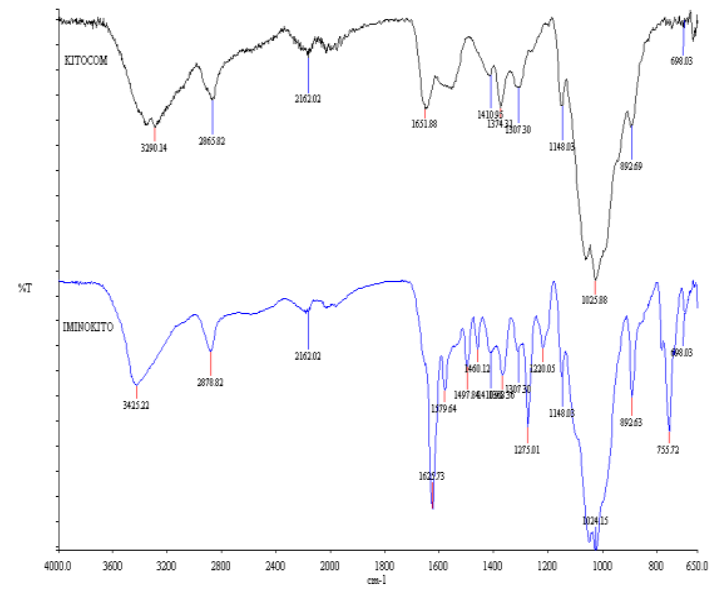

Fig. 1: Infrared spectra of Post-neutralised iminochitosan nanofiber (KITOCOM) and iminochitosan nanofiber (IMINOKITO).

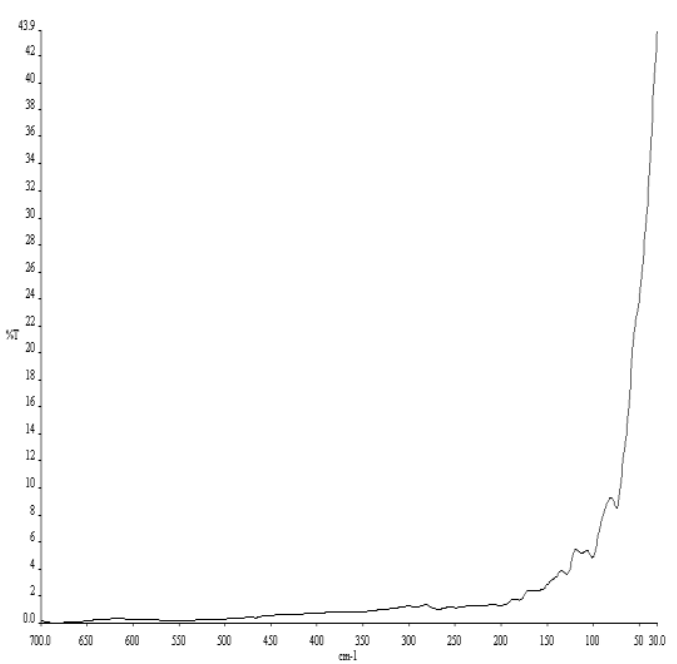

Fig. 2: Far IR region of $\mathrm{Cu}$ crown nanofiber.

The surface morphology of the nanofiber mat was studied (using a scanning electron microscope (SEM)) to have a clear insight into the formation of the fiber (Figure 3). Since fiber formation and morphologies are dependent on the properties of the polymer solution[16], the Schiff base chitosan was dissolved in TFA to form electrospinnable solution aided by sudden freezing and thawing. The optimal spinning parameters which gave bead-free nanofibers in the range $70-200 \mathrm{~nm}$, from $6 \mathrm{wt} \%$ solution are $24 \mathrm{kV}$ voltage, $15 \mathrm{~cm}$ working distance and 0.9 $\mathrm{mLh}^{-1}$. 


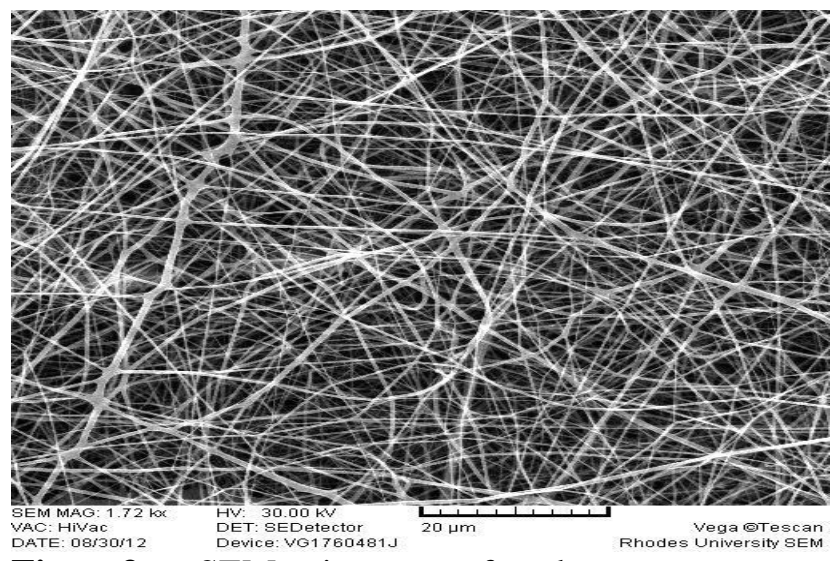

Fig. 3: SEM image of electrospun iminochitosan.

According to the thermogram, the nanofiber mat shows a high degree of stability to change in temperature (Figure 4). The nanofiber show little change in weight loss over a wide range of temperature. There was an observed weight loss around $100{ }^{\circ} \mathrm{C}$, which can be attributed to the removal of adsorbed water molecules. The second weight loss occurred at $320{ }^{\circ} \mathrm{C}$ ascribed to the decomposition of the polysaccharide chain [17].

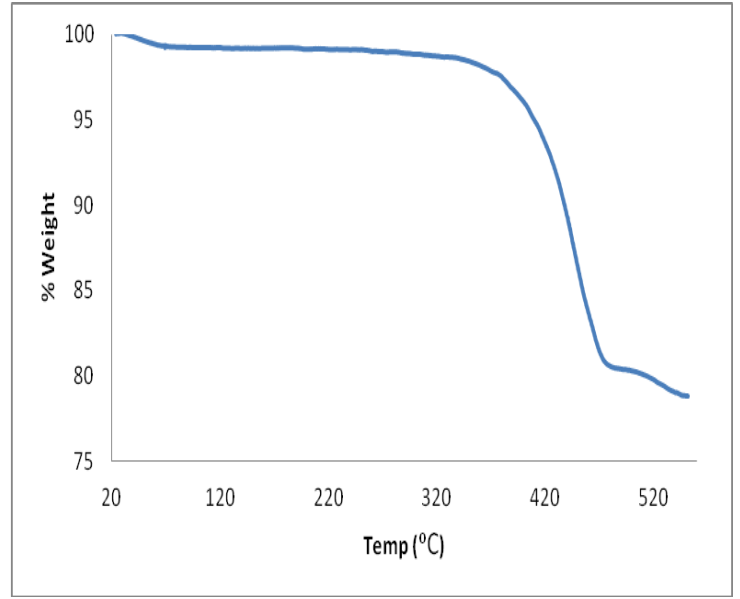

Fig. 4: TGA curve of copper crown nanofiber under nitrogen atmosphere

In addition, a differential scanning calorimetry (DSC) curve of the nanofiber shows distinct deviations from the usual step like changes in heat flow. A broad endothermic peak in the range of $100-150{ }^{\circ} \mathrm{C}$ was observed and this is related to the evaporation of water molecules in the nanofiber. The area and position of the endothermic peaks was outsized at higher temperature suggesting that the nanofiber has a high water holding capacity and strong interaction with water molecules. The exothermic peak expected clearly at $274{ }^{\circ} \mathrm{C}$ due to the decomposition of amino $(\mathrm{GlcN})$ has been significantly reduced meaning that it has been masked [18]. However, the exothermic peak around $390{ }^{\circ} \mathrm{C}$ can be attributed to the decomposition of the Nacetyl (GlcNAc) residues [19]. The last two exothermic peaks were observed clearly at $490{ }^{\circ} \mathrm{C}$ and $520{ }^{\circ} \mathrm{C}$ due to the thermal decomposition of imine $(\mathrm{C}=\mathrm{N})$ and benzene ring $\left(\mathrm{C}_{6} \mathrm{H}_{6}\right)$ residues, respectively [9].

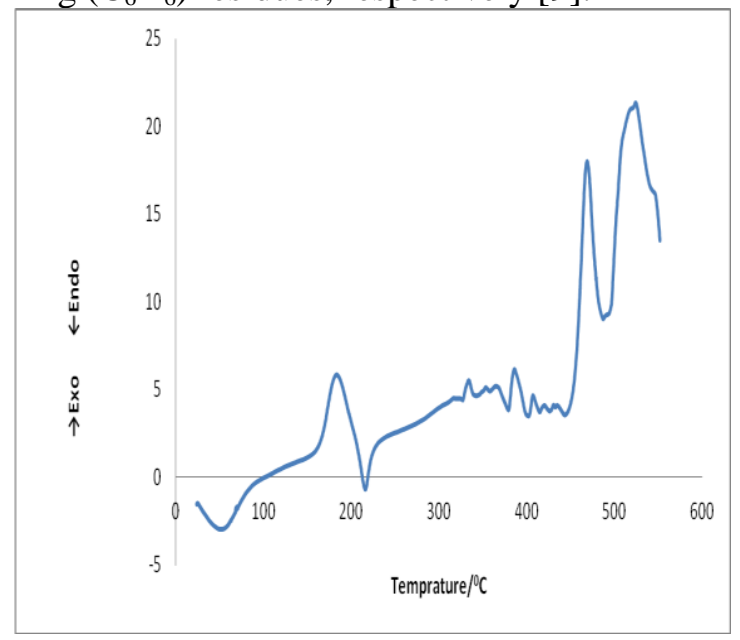

Fig. 5: DSC of the copper crown nanofiber

\subsection{Cyanide oxidation in the presence of copper-crown nanofiber}

Figure 6 compares cyanide oxidation efficiency of copper-crown nanofiber with only copper catalyst. Copper-crown nanofiber obviously displayed improved oxidation of cyanide (100\% after 20 mins) than only $\mathrm{Cu}$ catalyst ( $39 \%$ after $40 \mathrm{~min})$ in the presence of $\mathrm{H}_{2} \mathrm{O}_{2}$ oxidant and initial cyanide concentration $\left(\mathrm{C}_{\mathrm{o}}\right)$ of $100 \mathrm{mg} / \mathrm{L}$. The cyanide oxidation kinetics for copper-crown nanofiber displayed a two-phase profile, a rapid initial stage ( $\leq 10 \mathrm{~min}$ ) followed by a gradual reduction for 20 mins. By employing pseudo-first order reaction kinetics, the initial cyanide oxidation could be described by the model (Equation 3) [7, 18]. 
$d \frac{\left[C N^{-}\right]}{d t}=-K_{S A} a_{S} P_{m}\left[C N^{-}\right]=-K_{o b s}\left[C N^{-}\right]$

Where $\left[\mathrm{CN}^{-}\right]$is the aqueous cyanide concentration $(\mathrm{mg} / \mathrm{L})$ at time $\mathrm{t}(\mathrm{min}) . \mathrm{K}_{\mathrm{SA}}$ is the specific reaction rate constant based on the surface area of the nanofiber $(\mathrm{L} / \mathrm{min}$. $\left.\mathrm{m}^{2}\right), \mathrm{a}_{\mathrm{s}}$ is the specific surface area of the nanofiber $\left(\mathrm{m}^{2} / \mathrm{g}\right), \quad p_{\mathrm{m}}$ is the mass concentration of the nanofiber $(\mathrm{g} / \mathrm{L})$ and $\mathrm{K}_{\mathrm{obs}}$ is the observed pseudo-first-order rate constant $\left(\mathrm{min}^{-1}\right)$. Therefore, using equation 3 , the rate constants can then be determined by fitting the rate expression to the experimental data. The value of $k_{\text {obs }}$ was $0.236 \mathrm{~min}^{-1}$ for the $56.3 \mathrm{mg}$ copper-crown nanofiber and 0.015 $\mathrm{min}^{-1}$ for non- supported copper catalyst suggesting the copper-crown nanofiber to be 16 times greater than the oxidation with copper catalyst only. This marked difference can be attributed to increased specific surface area of the resultant nanofiber which greatly enhanced the surface reactivity of the catalyst.

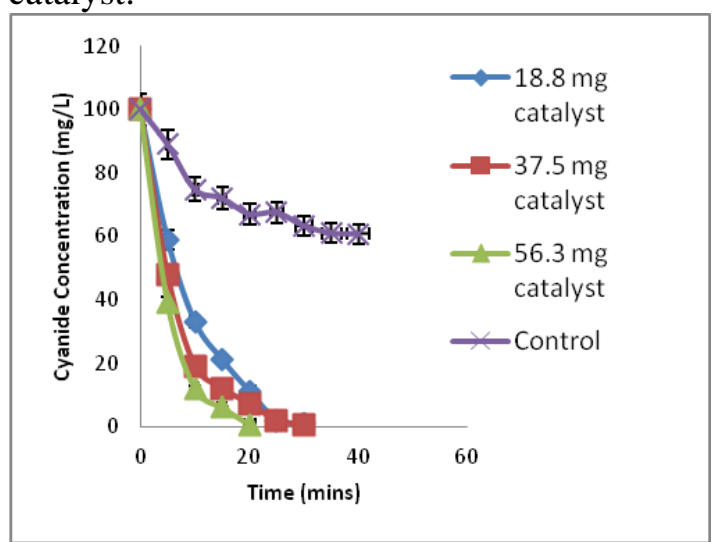

Fig. 6: Catalytic oxidation of aqueous cyanide solution in the presence of coppercrown nanofiber of different sizes (Values were represented as the mean of 3 readings i.e $n=3$ ).

\subsection{Effect of $\mathrm{H}_{2} \mathrm{O}_{2} / \mathrm{CN}$ mole ratio}

In order to accomplish optimal cyanide oxidation, the ratio of the oxidant to cyanide was investigated (Fig. 7). At the $\mathrm{H}_{2} \mathrm{O}_{2} / \mathrm{CN}^{-}$ ratio of 2.0 in the presence of the coppercrown nanofiber, the oxidation profile displayed the highest efficiency. This observation is in accord with the study of Kim et al.[20] which reported that cyanide removal efficiency and rate of reaction increased considerably with an increase in $\mathrm{H}_{2} \mathrm{O}_{2}$ concentration.

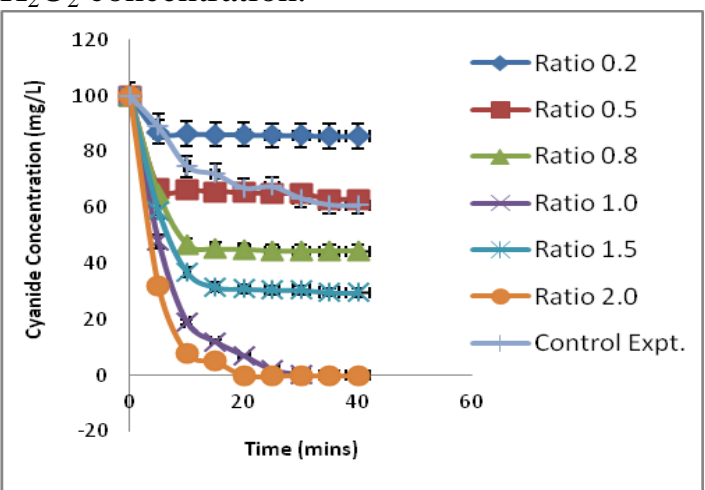

Fig. 7: Effects of $\mathrm{H}_{2} \mathrm{O}_{2} / \mathrm{CN}^{-}$ratio on the catalytic oxidation of aqueous cyanide solution by hydrogen peroxide in the presence of copper-crown nanofiber (Values were represented as mean of 3 readings i.e $n$ $=3$ ).

\subsection{Effect of $\mathrm{pH}$ on cyanide oxidation}

Figure 8 shows the plot of the residual concentration of cyanide at various time intervals and $\mathrm{pH}$. At $\mathrm{pH} 7$, almost $100 \%$ cyanide catalytic oxidation has been attained within the first $10 \mathrm{~min}$ of the reaction. However, at lower $\mathrm{pH}$, the percentage conversion was reduced. This observation could be attributed to the fact that at acidic $\mathrm{pH}$, cyanide is mainly present in the form of $\mathrm{HCN}$ gas which is difficult to oxidize [7]. Also, the percentage conversion was further reduced at higher $\mathrm{pH}$, which is largely due to the decomposition of hydrogen peroxide in an alkaline solution [21].

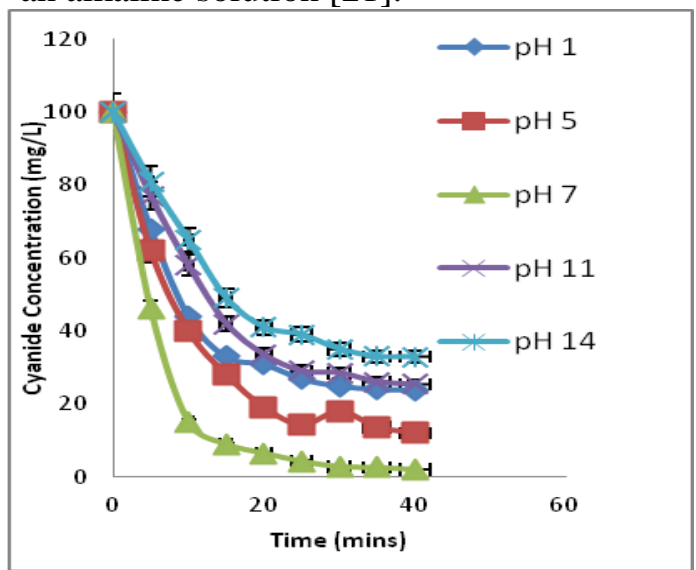

Fig. 8: Effect of $\mathrm{pH}$ on the catalytic oxidation of aqueous cyanide solution by hydrogen peroxide in the presence of copper-crown 
nanofiber (Values were represented as mean of 3 readings i.e $n=3$ ).

3.5 Effect of temperature on cyanide oxidation

Figure 9 shows the effects of temperature on the oxidation of cyanide by the copper-crown nanofiber. The temperature effect was studied by the oxidation of $100 \mathrm{mg} / \mathrm{L}$ cyanide solution using $160 \mathrm{mg} / \mathrm{L} \mathrm{H}_{2} \mathrm{O}_{2}$ in the presence of $37.5 \mathrm{mg} / \mathrm{L}$ catalyst mix and was conducted over temperature range from 18 to $50{ }^{\circ} \mathrm{C}$. It was observed that increase in temperature generally affected the catalytic oxidation of cyanide. At the optimum temperature of 50 ${ }^{\circ} \mathrm{C}$, over $80 \%$ cyanide conversion was achieved within the first $2 \mathrm{~min}$. This observation is in accord with Pak and Chang [6] where high temperature higher than $50{ }^{\circ} \mathrm{C}$ was required for complete oxidation of cyanide.

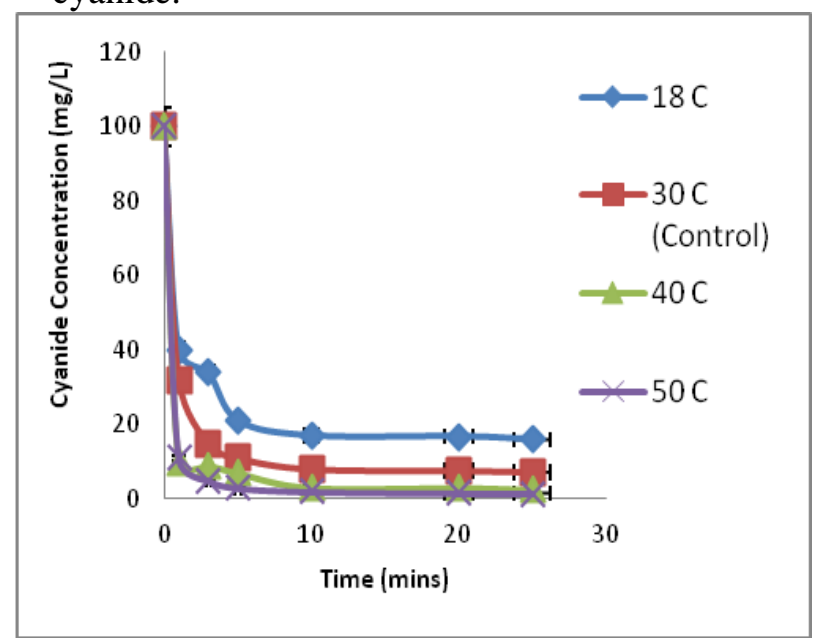

Fig. 9: Effect of temperature on the catalytic oxidation of aqueous cyanide.

3.6 Copper-crown nanofiber reusability studies

Figure 10 shows the results obtained for the reusability of the copper-crown nanofiber catalyst for 5 runs under the same conditions. After the fifth cycle of catalytic oxidation of cyanide, the percentage efficiency yield is more than $65 \%$. This is an indication that the catalyst mix could be reused for at least 5 cycles without much loss in its activity. Obviously, this confers a better advantage above other heterogeneous systems previously used for cyanide conversion.

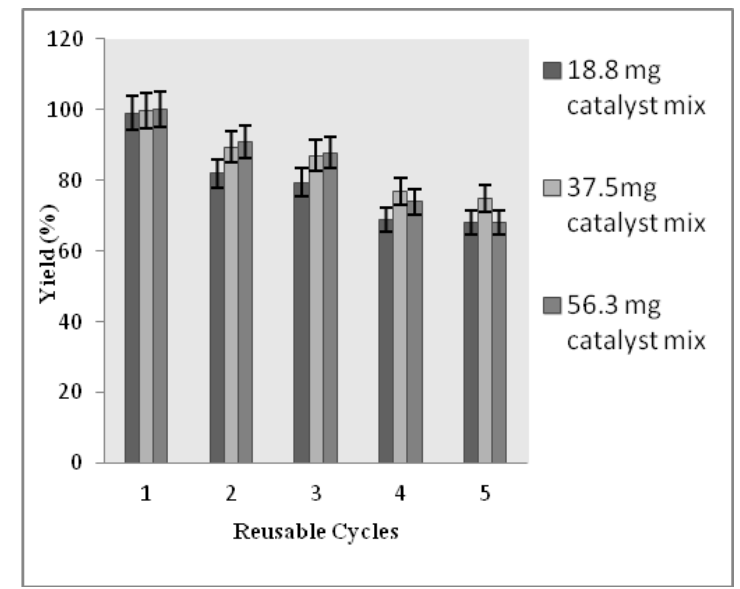

Fig. 10: The reusability tests of the different weights of copper-crown nanofiber for the catalytic oxidation of aqueous cyanide by hydrogen peroxide (Values were represented as mean of 3 readings i.e $n=3$ ).

\subsection{CONCLUSION}

The oxidation of aqueous cyanide solution using hydrogen peroxide was greatly enhanced when copper-crown chitosan nanofiber was used as a catalyst. The observed pseudo-first-order rate constant $\left(\mathrm{k}_{\mathrm{obs}}\right)$ for the oxidation process with coppercrown nanofiber was found to be 16 times greater when compared with oxidation using only copper catalyst. The catalytic oxidation in the presence of copper-crown nanofiber was observed to be dependent on the reaction conditions of $\mathrm{pH}$, temperature and $\mathrm{H}_{2} \mathrm{O}_{2} / \mathrm{CN}^{-}$ ratio. The reusability of the copper-crown nanofiber catalyst confers a special advantage on this heterogeneous system making it potentially viable for commercial applications.

\section{Acknowledgement}

This work was supported by the Department of Chemistry, University of Agriculture, Abeokuta, Nigeria and Chemistry Department, Rhodes University, Grahamstown, South Africa.

\section{References}

[1].World Health Organization (2007). Cyanide in drinking-water, Background 
document for development of WHO Guidelines for Drinking-water Quality, WHO Press, Switzerland, WHO/SDE/WSH/07, 01/2 1-18.

[2]. B. Hosetti, P. Dube, and A. Shwetha, A.(2011). Changes in the freshwater fish Catla catla under copper cyanide intoxication, International Journal of Pharmaceutical \& Biological Archives, 2, 874-879.

[3]. M. Botz, (2001). Overview of cyanide treatment methods, Mining environmental management, Mining Journal Ltd., London, UK, 2-17.

[4]. L. Tae-Yoon, K. Young-Shik, and K. Dong-Su, (2004). Oxidative treatment of cyanide in waste water using hydrogen peroxide and homogenous catalyst. Journal of Environmental Science and health, part A: Toxic/Hazardous Subtances and Environmental Engineering, 39, 787-801.

[5]. M. Kitis, E. Karakaya, N. Yigit, G. Civelekoglu, and A. Akcil, (2005). Heterogeneous catalytic degradation of cyanide using copper-impregnated pumice and hydrogen peroxide, Water Research, 39, 1652-1662"

[6].D. Pak, and W. Chang, (1997). Oxidation of Aqueous Cyanide solution using hydrogen peroxide in the presence of heterogenous catalyst, Environmental Technology, 18, 557-561.

[7]. M. Sarla, M. Pandit, D. Tyagi, and J. Kapoor, (2004). Oxidation of cyanide in aqueous solution by chemical and photochemical process, Journal of Hazardous Materials B, 116, 49-56.

[8]. S. Adewuyi, J. Jacob, O. Olaleye, T. Abdulraheem, J. Tayo, and F. Oladoyinbo, (2016).

Chitosan-bound pyridinedicarboxylate $\mathrm{Ni}(\mathrm{II})$ and $\mathrm{Fe}(\mathrm{III})$ complex biopolymer films as waste water decyanidation agents, Carbohydrate Polymers, 151, 1235-1239.

[9]. N. Sanyaolu, S. Adewuyi, T. Shittu, C.

Eromosele, and N. Torto, (2018). Synthesis and Characterization of Electrospunned Chitosan Based Copper Nanofiber, AASCIT Journal of Chemistry, 4(2), 7-17

[10]. K. El-Tahlawy, E. Abdelhaleem, S. Hudson, and A. Hebeish, (2007). Acylation of iminochitosan: Its effect on blending with cellulose acetate, Journal of Applied Polymer Science, 104, 727-734.

[11]. R. Nawalakhe, S. Hudson, A. Seyam, A. Waly, N. Abou-Zeid, and H. Ibrahim, (2012). Development of electrospun iminochitosan for improved wound healing application, Journal of Engineering Fibers and Fabrics, 7, 47-55.

[12].S. Haider, and S. Park, (2009). Preparation of the electrospun chitosan nanofibers and their applications to the adsorption of $\mathrm{Cu}$ (II) and $\mathrm{Pb}$ (II) ions from an aqueous solution, Journal of Membrane Science, 328, 90-96.

[13]. Z. Ye, Y. Alsyouri, S. Zhu, and Y. Lin, (2003). Catalystimpregnation and ethylene polymerization with mesoporous particle supported nickel-diimine catalyst, Polymer, 44, 969-980.

[14]. F. Xie, and B. Dreisinger, (2010). Copper solvent extraction from alkaline cyanide solution with guanidine extractant LIX 7950, Transaction of Nonferous Metals Society of

China, 20, 1136-1140.

[15]. G. Drochioiu, (2002). Fast and highly selective determination of cyanide with 2,2dihydroxy-1,3 indanedione, Talanta, 56, 1163-1165.

[16]. D. Hussain, F. Loyal, A. Greiner, and J. Wendorff,(2010). Structure property correlations for electrospun nanofiber nonwovens. Polymer, 51, 3989-3997.

[17]. Y. Nam, H. Won, I. Daewoo, and M Samuel, (2010). Effects of the degree of deacetylation on the thermal decomposition of chitin and chitosan nanofibers. Carbohydrate Polymers, 80, 291-295.

[18]. K. Sakurai, T. Maegawa, and T. Takahashi, (2000). Glass transition temperature of chitosan and miscibility of chitosan/poly( $N$-vinyl pyrrolidone) blends, Polymer, 41, 7051-7056

[19]. P. Sangsanoh, and P. Supaphol, (2006). Stability improvement of electrospun chitosan nanofibrous membranes in neutral or weak basic aqueous solutions. Biomacromolecules, 7, 2710-2714.

[20]. Y. Kim, T. Qureshi, and K. Min, (2008). Application of advanced oxidation 
processes for the treatment of cyanide containing effluent, Environmental Technology, 24, 1269-1276

[21]. A. De, B. Chaudhuri, S. Bhattacharjee, and B. Dutta, (1999). Estimation of $\mathrm{OH}^{\circ}$ Radical reaction rate constant for phenol using $\mathrm{UV} / \mathrm{H}_{2} \mathrm{O}_{2}$ photooxidation. Journal of Hazardous Materials, 64, 91-104. 